\title{
ON THE OSCILLATORY PROPERTIES OF THE SOLUTIONS OF A CLASS OF INTEGRO-DIFFERENTIAL EQUATIONS OF NEUTRAL TYPE
}

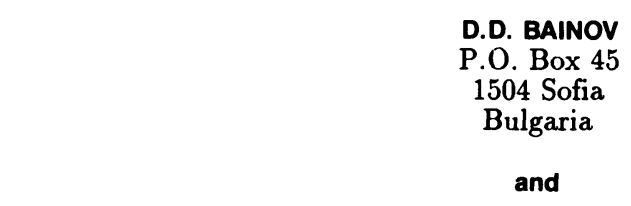

D.D. BAINOV

Box 45

1504 Sofia

and

A.D. MYSHKIS

PLOVDIV UNIVERSITY

"PAISSII HILENDARSKI"

\begin{abstract}
A.I. ZAHARIEV
MOSCOW INSTITUTE OF

RAILWAY TRANSPORT

ENGINEERING
\end{abstract}

(Received JAnuary 17, 1990 and in revised form September 6, 1990)

ABSTRACT. In the present paper the oscillatory properties of the solutions of the equation

$$
[(L x)(t)]^{(n)}+\int_{I_{t}} K(t, s, x(s)) d s=0
$$

are investigated where $n \geq 1, L$ is an operator of the difference type, $I_{t} \subset \mathbf{R}, K: D_{K} \rightarrow \mathbf{R}$, $D_{K} \subseteq \mathbb{R}^{3}, x:\left[\alpha_{x}, \infty\right] \rightarrow \mathbb{R}$. Under natural conditions imposed on $L, I_{t}$ and $K$ it is proved that for $n$ even all ultimately nonzero solutions oscillate and for $n$ odd they either oscillate or tend to zero as $t \rightarrow \infty$.

KEY WORDS AND PHRASES: Integral equations, operator differential equations, oscillatory and non-oscillatory solutions.

1980 AMS SUBJECT CLASSIFICATION CODES. 34C, 45N05, 45G05.

\section{INTRODUCTION.}

In the last 20 years, the oscillatory and asymptotic theory of functional differential equations marked a rapid development. This development, in particular, is due to the fact that by means of such equations many phenomena and processes in the field of natural sciences and technology are simulated. Among the functional differential equations, the equations of neutral type occupy a particular place in relation to the circumstance that, on the one hand, they have a considerably more complex structure and, on the other hand, many of their properties have no analogous with the other functional differential equations. This explains, to a certain extent, the small amount of work dedicated to the investigation of the asymptotic and oscillatory properties of the solutions of functional differential equations of neutral type. (Norkin [1], Myshkis, et. al. [2,3,4], and Zahariev and Bainov $[5,6])$.

In [2] (see also [4]) a general method for the proof of the oscillatory properties of the solutions of functional differential equations of the neutral type was suggested. In particular, this method 
was applied to equations of the type

$$
[x(t+\tau)+\lambda x(t)]^{(n)}+\int_{t}^{t+T} K(t, s, x(s)) d s=0
$$

where $n \geq 1$ and $t, \lambda, T$ are positive constants.

Analogous results were obtained in Bainov, Myshkis, and Zahariev [7] for a more general class of equations which have the form

$$
[x(t+\tau)+\lambda x(t)]^{(n)}+\int_{\alpha(t)}^{\beta(t)} K(t, s, x(s)) d s=0, \alpha(t) \leq \beta(t)
$$

The main problem was to find conditions which must be satisfied by the functions $\alpha$ and $\beta$ so that the general method could be applied to equation (1.2). This required the use of essentially new arguments and the results obtained were announced without proofs in [4].

In the present paper these results are extended to a class of equations of the form

$$
[(L x)(t)]^{(n)}+\int_{I_{t}} K(t, s, x(s)) d s=0,
$$

where $I_{t}$ is a measurable subset of $\mathbb{R}$ and $L$ is an operator of the difference type (see, for instance, equality (2.3) below). It is immediately seen that equation (1.2) is a particular case of equation (1.3) where $T_{t}=[\alpha(t), \beta(t)]$ but, independent of this, some of the results obtained in the present paper are new also for equations of the form (1.2).

2. BASIC CONCEPTS AND MAIN RESULTS.

DEFINITION 2.1. We shall say that the property $Q$ which depends on the parameter $t$ is fulfilled if it is fulfilled for all sufficiently large values of $t \in \boldsymbol{R}$.

DEFINITION 2.2. The function $\varphi:\left[\alpha_{\varphi}, \infty\right) \rightarrow \mathbb{R}$ is said to be with constant sign if $\varphi(t) \geq 0$ $(\varphi(t) \leq 0)$ for $t \in\left[\alpha_{\varphi}, \infty\right)$. In particular, the zero function $\varphi(t) \equiv 0$ can be regarded as a nonnegative as well as nonpositive function with constant sign.

DEFINITION 2.3. The function $\varphi:\left[\alpha_{\varphi}, \infty\right) \rightarrow \mathbb{R}$ will be called oscillating if it is not ultimately with constant sign.

By $A C^{n}[\alpha, \infty)\left(A C^{-1}[\alpha, \infty)\right), n \geq 0$, we shall denote the space of functions $\varphi:[\alpha, \infty) \rightarrow \mathbf{R}$ with locally absolutely continuous derivatives up to the $n$-th order (locally summable).

Consider the operator-differential equation

$$
[(L x)(t)]^{(n)}+(F x)(t)=0
$$

We assume that the operators $L$ and $F$ are defined on some non-empty set $D$ of functions $\varphi \in A C^{n-1}\left[\alpha_{\varphi}, \infty\right)$ and for each $\varphi \in D$ we have $L \varphi \in A C^{n-1}\left[\beta_{\varphi}, \infty\right), F \varphi \in A C^{-1}\left[\gamma_{\varphi}, \infty\right)$, $\beta_{\varphi}, \gamma_{\varphi} \in \mathbf{R}$.

DEFINITION 2.4. A function $x \in D$ will be called an ultimate solution of equation (2.1) if it turns it into identity for almost all $t \geq t_{x}, t_{x} \in \mathbb{R}$.

DEFINITION 2.5. We shall say that equation (1.3) has the property $A$ if each of its solutions $x$ for $n$ even either oscillates or is ultimately zero and for $n$ odd either oscillates or $x(t)$ and $(L x)(t)$ tend to zero as $t \rightarrow \infty$. 
THEOREM 1 [3]. Let the operators $L$ and $F$ satisfy the following conditions:

1L. If the function $\varphi \in D$ is ultimately with constant signs, then the function $L \varphi$ is ultimately with constant sign as well. Moreover, $\varphi$ and $L \varphi$ have the same sign, i.e., if ultimately $\varphi(t) \geq 0$ $(\varphi(t) \leq 0)$, then ultimately $(L \varphi)(t) \geq 0$ as well $((L \varphi)(t) \leq 0)$.

$1 F$. If the function $\varphi \in D$ is ultimately with constant sign, then the function $F \varphi$ is ultimately almost everywhere with constant sign and $\varphi$ and $F \varphi$ have the same sign.

2L. If the function $\varphi \in D$ is ultimately with constant sign and $L \varphi$ is ultimately zero, then $\varphi$ is ultimately zero.

3L. If $n$ is odd, $\varphi \in D$ is ultimately with constant sign and $\lim _{t \rightarrow \infty}(L \varphi)(t)=0$, then $\lim _{t \rightarrow \infty} \varphi(t)=0$.

1LF. If the function $\varphi \in D$ is ultimately with constant sign and $\liminf _{t \rightarrow \infty}|(L \varphi)(t)|>0$, then

$$
\int^{\infty}|(F \varphi)(t)| d t=\infty
$$

Then equation (2.1) has the property $A$.

REMARK 2.1. We shall note that the formulation of Theorem 2.1 is an immediate generalization of Theorem 4 from [3] and the proof repeats literally the proof of Theorem 4 from [3].

Let the operator $L$ from equation (1.3) be as in equation (2.1) and let the function $K: D_{K} \rightarrow \mathbb{R}$ where $D_{K}$ is a measurable subset of $\mathbb{R}^{3}$. By $\&$ we denote some set of unbounded above and measurable subsets of $\mathbb{R}$.

We shall assume that the function $K$ and the set $I_{t}$ satisfy the following conditions:

1I. There exists a number $\gamma \in \mathbb{R}$ such that the set $I_{t}$ is defined and non-empty for $t \in[\gamma, \infty)$, the set $Q=\left\{(t, s) \in \mathbb{R}^{2} \mid t \in[\gamma, \infty), s \in I_{t}\right\}$ is measurable and for $t>\gamma$ the following relation holds:

$$
\int_{\gamma}^{t} \text { meas } I_{\tau} d \tau<\infty
$$

REMARK 2.2. In order for the function $K_{\varphi}$ to be measurable, it suffices for the function $K$ to satisfy the Carathéodory conditions.

THEOREM 2.1. Let the following conditions be fulfilled:

1. Conditions $1 \mathrm{~L}-3 \mathrm{~L}, 1 \mathrm{I}$ and $1 \mathrm{~K}$ hold.

2. For each ultimately with constant signs function $\varphi \in D$ such that $\liminf _{t \rightarrow \infty}|(L \varphi)(t)|>0$, there exists a set $E_{\varphi} \in \mathcal{8}, E_{\varphi} \subseteq\left[\alpha_{\varphi}, \infty\right)$ and a constant $C_{\varphi}>0$ such that $|\varphi(t)| \geq C_{\varphi}$ for $t \in E_{\varphi}$.

3. There exist numbers $t_{k}, s_{k} \in \mathbf{R}$ such that

$$
\operatorname{sgn} K(t, s, u)=\operatorname{sgn} u
$$

for $t \geq t_{k}, s \geq s_{k},(t, s, u) \in D_{K}$.

4. For each constant $c>0$ the inequality $\liminf _{t, s \rightarrow \infty}|K(t, s, n)|>0,|N| \geq c,(t, s, n) \in D_{K}$ holds if its left-hand side makes sense.

5. $\lim _{t \rightarrow \infty}\left(\inf I_{t}\right)=\infty$. 
6. For each set $E \in \&$ the following relation holds

$$
\int_{E} \operatorname{meas}\{t \mid(t, s) \in Q\} d s=\infty
$$

Then equation (2.1) has the property $A$.

PROOF. It is immediately seen that conditions 3 and 5 of Theorem 2.1 imply condition $1 F$ of Theorem 1 for the operator

$$
(F \varphi)(t):=\int_{I_{t}} K(t, s, \varphi(s)) d s
$$

We shall prove that for the operators $L$ and $F$ from (1.1) condition 1LF is fulfilled.

Let $\varphi \in D$ and assume, for the sake of definiteness, that $\varphi(t) \geq 0$ for $t \in\left[\delta_{\varphi}, \infty\right]$ and $\liminf _{t \rightarrow \infty}(L \varphi)(t)>0$. By condition 2 of Theorem 2.1 there exists a set $E_{\varphi} \in \&$ and a constant $C_{\varphi}>0$ such that $\varphi(t) \geq C_{\varphi}$ for $t \in E_{\varphi}$. From conditions 3 and 4 of Theorem 2.1 it follows that there exists a number $\varepsilon_{\varphi} \geq s_{k}$ such that the following inequality holds

$$
a_{\varphi}:=\liminf _{\substack{u \geq C_{\varphi} \\ t, s \geq \varepsilon_{\varphi}}} K(t, s, u)>0,(t, s, u) \in D_{K} .
$$

Moreover, from condition 2.2 of Theorem 2.1 it follows that there exists a number $\xi_{\varphi} \geq t_{k}$ such that for $t \geq \xi_{\varphi}$ the inequality inf $I_{t} \geq \max \left(\varepsilon_{\varphi}, \alpha_{\varphi}\right)$ holds.

It remains to prove that

$$
\int_{\xi_{\varphi}}^{\infty}\left(\int_{I_{t}} K(t, s, \varphi(s)) d s\right) d t=\infty
$$

The Fubini Theorem and condition 6 of Theorem 2.1 imply the inequalities

$$
\begin{gathered}
\int_{\xi_{\varphi}}^{\infty}\left(\int_{I_{t}} K(t, s, \varphi(s)) d s\right) d t=\int_{\varepsilon_{\varphi}}^{\infty}\left\{t \in\left[\xi_{\varphi}, \infty\right) \mid S \in I_{t}\right\} \\
\left.\quad \geq \int_{E_{\varphi} \cap\left[\varepsilon_{\varphi}, \infty\right)} K_{\varphi}(t, s) d t\right) d s \\
\quad\left(\int_{\left.t \in\left[\xi_{\varphi}, \infty\right) \mid s \in I_{t}\right\}} a_{\varphi} d t\right) d s \\
a_{E_{\varphi} \cap\left[\varepsilon_{\varphi}, \infty\right)} \operatorname{meas}\left\{t \in\left[\xi_{\varphi}, \infty\right) \mid s \in I_{t}\right\} d s=\infty .
\end{gathered}
$$

This completes the proof of Theorem 2.1.

REMARK 2.3. Condition 4 of Theorem 2.1 can be weakened which will extend the scope of its applications, namely conditions 4 and 6 of Theorem 2.1 can be replaced by a "combined" condition of the following form: for any element $E \in \&$ and any constant $c>0$ the following relation holds

$$
\int_{E}\left\{\int_{\{t \mid(t, s) \in Q\}} \inf _{|u| \geq c}|K(t, s, u)| d t\right\} d s=\infty .
$$


Analogously, the respective assumptions in Theorems 3 and 4 and in Corollary 2.1 can be weakened.

Consider the operator

$$
(L \varphi)(t):=\sum_{i=1}^{m} a_{i}(t) \varphi\left(h_{i}(t)\right), \quad t \in\left(t_{o}, \infty\right)
$$

where $m \geq 1, a_{i} \in C\left[t_{o}, \infty\right), \sup _{t \in\left[t_{o}, \infty\right)}\left|a_{i}(t)\right|<\infty, h_{i} \in C\left[t_{o}, \infty\right), i=\overline{1, m}$.

Now we shall consider the question what properties the totality of sets 8 should have so that the operator $L$ defined by equality (2.4) should satisfy condition 2 of Theorem 2.1 .

LEMMA 2.1. Let the following conditions be fulfilled:

1. There exists a constant $\tau>0$ such that

$$
\sup _{t \in\left[t_{o}, \infty\right]}\left|h_{i}(t)-t\right| \leq \tau, \quad i=\overline{1, m}
$$

2. There exists a constant $l>0$ such that for each closed set $B \subseteq\left[t_{o}, \infty\right]$ the following inequality holds

$$
\text { meas } h_{i}(B) \geq l \text { meas } B, \quad i=\overline{1, m} \text {. }
$$

3. The totality $\&$ consists of all measurable sets $E \subseteq R$ satisfying the estimate

$$
\operatorname{meas}(E \cap[t, t+3 \tau]) \geq \min \left(3 \tau, \frac{l \tau}{m}\right), \text { for } t \geq \alpha_{E}
$$

Then, if we set $D=C\left[t_{o}, \infty\right)$, the operator $L$ defined by equality (2.4) satisfies condition 2 of Theorem 2.1.

PROOF. Let $\varphi \in C\left[t_{o}, \infty\right)$ be an arbitrary function with ultimately constant sign and assume that $\left|a_{i}(t)\right| \leq A, i=\overline{1, m}, A>0,|(L \varphi)(t)| \geq C>0$ for $t \in\left[\delta_{\varphi}, \infty\right)$. (From condition 1 of Lemma 2.1 it follows that for $t \geq t_{o}+\tau$ the right-hand side of (2.4) is defined.)

Set $C_{\varphi}=\frac{C}{m A}, E_{\varphi}=\left\{t \geq \delta_{\varphi}|\varphi(t)| \geq C_{\varphi}\right\}$ and $\alpha_{E}=\delta_{\varphi}$. For $t \geq \delta_{\varphi}$ we obtain

$$
\bigcap_{i=1}^{m}\left\{s \in[t+\tau, t+2 \tau] \mid h_{i}(s) \notin E_{\varphi}\right\}=\emptyset,
$$

which implies

Hence

$$
\sum_{i=1}^{m} \text { meas }\left\{s \in[t+\tau, t+2 \tau] \mid h_{i}(s) \in E_{\varphi}\right\} \geq \tau .
$$

$$
\sum_{i=1}^{m} l^{-1} \text { meas }\left\{h_{i}([t+\tau, t+2 \tau]) \cap E_{\varphi}\right\} \geq \tau .
$$

Inequality (2.6) implies inequality (2.5) for $E=E_{\varphi}$.

REMARK 2.4. If for $i=\overline{1, m}$, the inequality $h_{i}(t) \geq t\left(h_{i}(t) \leq t\right)$ holds, i.e., all transformed arguments are of forestalling (retarding) type, then in estimate (2.5) we can replace $3 \tau$ by $2 \tau$.

REMARK 2.5. If meas $\left\{h_{i}([t, t+\tau]) \cap h_{j}([t, t+\tau])=0\right.$ for $t \in\left[t_{o}, \infty\right), i, j=\overline{1, m}, i \neq j$, then from inequality (2.6) it follows that in the right-hand side of estimate (2.5) the number $\frac{l \tau}{m}$ can be 
replaced by $l \tau$. This assertion still holds if we replace $3 \tau$ by $2 \tau$ and all transformed arguments $h_{i}(t), i=\overline{1, m}$, are either of forestalling or of retarding type only. In particular, this is satisfied for the operator $L$ in equation (1.1).

REMARK 2.6. If the functions $h_{i} \in A C\left[t_{o}, \infty\right), i=\overline{1, m}$, then condition 2 of Lemma 2.1 is equivalent to the following condition:

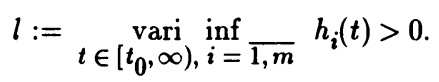

LEMMA 2.2. Let the following conditions be fulfilled:

1. The set $E \subseteq R$ is measurable.

2. There exist constants $p, q, \alpha_{E} \in R, p \geq q>0$ such that for $t \geq \alpha_{E}$ the following inequality holds

$$
\text { meas }(E \cap[t, t+p]) \geq q \text {. }
$$

Then for each measurable function $f:\left[\alpha_{E}, \infty\right) \rightarrow[0, \infty)$ the following inequality is satisfied

PROOF.

$$
\int_{E \cap\left[\alpha_{E}, \infty\right)} f(s) d s \geq q p^{-1} \int_{\alpha_{E}}^{\infty} \inf _{s \leq \sigma \leq s+2 p} f(\sigma) d s
$$

$$
\begin{aligned}
\int_{E \cap\left[\alpha_{E}, \infty\right)} f(s) d s & \geq \sum_{i=1}^{\infty} \int_{E \cap\left[\alpha_{E}+i p, \alpha_{E}+(i+1) p\right]} f(s) d s \\
& \geq q \sum_{i=1}^{\infty} \operatorname{linf}_{\alpha^{+i p} \leq \sigma \leq \alpha_{E}+(i+1) p} f(\sigma) \\
& \geq q p^{-1} \sum_{i=1}^{\infty} \int_{\alpha_{E}+(i-1) p}^{\alpha_{E}+i p} s \leq \inf _{s+2 p} f(\sigma) d s \\
& =q p^{-1} \int_{\alpha_{E}}^{\infty} s \leq \sigma \leq s+2 p
\end{aligned}
$$

Consider the equation

$$
\left[\sum_{i=1}^{m} a_{i}(t) x\left(h_{i}(t)\right)\right]^{(n)}+\int_{I_{t}} K(t, s, x(s)) d s=0, t \geq t_{o}
$$

where $m, n \geq 1, a_{i} \in A C^{n-1}\left[t_{o}, \infty\right), \sup _{t \geq t_{o}}\left|a_{i}(t)\right|<\infty$ and $h_{i} \in A C^{n}\left[t_{o}, \infty\right), i=\overline{1, m}$.

THEOREM 2.2. Let the following conditions be fulfilled:

1. Conditions 1 and 2 of Lemma 2.1 hold.

2. Conditions $1 \mathrm{I}, 1 \mathrm{~K}$ and conditions $3-5$ of Theorem 2.1 hold.

3. All functions $a_{i}(t)$ are ultimately nonnegative, $a_{1}(t)$ has no intervals of zeros and for $n$ odd the relation $\lim _{t \rightarrow \infty} \inf _{1} a_{1}(t)>0$ holds.

4. The following relation holds

$$
\int_{s \leq \sigma \leq s+6 \tau}^{\infty} \underset{v a r i}{\inf } \operatorname{meas}\{t \mid(t, \sigma) \in Q\} d s=\infty
$$


Then equation (2.1) has the property $\mathrm{A}$.

PROOF. It is immediately seen that for the operator $L$ defined by equality (2.4) condition 3 of Theorem 2.2 implies conditions 1L-3L. Therefore, in order to apply Theorem 2.1 it remains to prove that condition 6 of Theorem 2.1 holds for any measurable set $E$ satisfying estimate (2.5). This is obtained immediately from Lemma 2.2 if we set $f(s)=$ meas $\{t \mid(t, s) \in Q\}$.

This completes the proof of Theorem 2.2.

REMARK 2.7. If all transformed arguments $h_{i}(t), i=\overline{1, m}$, are of the forestalling (retarding) type, then the number $6 \tau$ in relation (2.8) can be replaced by $4 \tau$.

REMARK 2.8. Set

$$
I_{t}=[\alpha(t), \beta(t)], t \in\left[t_{o}, \infty\right)
$$

where $\alpha$ and $\beta$ are continuous ultimately strictly increasing functions and $\alpha(\infty)=\infty$. Denote by $\bar{\alpha}$ and $\bar{\beta}$ the inverse functions to $\alpha$ and $\beta$ and assume that the function $\bar{\alpha}-\bar{\beta}$ is ultimately nondecreasing or ultimately non-increasing. Then relation (2.8) is satisfied if and only if the following relation holds

$$
\int_{t_{o}}^{\infty}[\beta(t)-\alpha(t)] d t=\infty
$$

Another sufficient condition for relation (2.8) to be satisfied when $I_{t}$ is of the form (2.9) is either the function $\alpha$ or the function $\beta$ to be ultimately Lipschitz continuous and, moreover, the following relations to be fulfilled $\limsup _{t \rightarrow \infty} \alpha(t)=\infty, \lim _{t \rightarrow \infty}[\beta(t)-\alpha(t)]>0$.

The last two conditions are satisfied, for instance, for the set $I_{t}$ in equation (1.1).

REMARK 2.9. We shall show that condition (2.8) is precise even for equations of the type (1.2).

Consider the equation

$$
[x(t+\tau)+\lambda x(t)]^{\prime}+\mu \int_{\alpha(t)}^{\beta(t)} x(s) d s=0,
$$

where $n=1 ; \tau, \mu$ are positive constants, $\lambda \in(0,1)$ and the functions $\alpha(t)$ and $\beta(t)$ satisfy the conditions formulated in Remark 2.8. Then conditions (2.8) and (2.10) are equivalent and if condition (2.10) holds, from Theorem 2.2 it follows that equation (2.11) has the property $A$.

Now let condition $(2.10)$ be not fulfilled, i.e.

$$
\int_{t_{o}}^{\infty}[p(t)-\alpha(t)] d t<\infty
$$

and choose a number $t_{1} \in\left[t_{o}, \infty\right)$ such that the following inequality should hold

$$
\lambda+\int_{t_{1}}^{\infty}[\beta(t)-\alpha(t)] d t<1 .
$$

Set $t_{2}:=\min \left(t_{1}, \min _{t \geq t_{1}+\tau} \alpha(t)\right)$ and denote by $Q$ the set of all continuous functions $x:\left[t_{2}, \infty\right) \rightarrow \mathbb{R}$ such that $\lim _{0 \rightarrow \infty} x(t)=1$. Introduce into the set $Q$ the usual (Chebyshev's) metric 
and define the operator $K$ by the equality

$(K x)(t)=\left\{\begin{array}{l}1+\lambda-\lambda x(t-\tau)+\mu \int_{t-\tau}^{\infty}\left(\int_{\alpha(\tau)}^{\beta(\tau)} x(s) d s\right) d \tau, t \in\left[t_{1}+\tau, \infty\right) \\ x(t)-x\left(t_{1}+\tau\right)+(\mathbb{K} x)\left(t_{1}+\tau\right), t \in\left[t_{2}, t_{1}+\tau\right) .\end{array}\right.$

It is immediately verified that $K Q \subseteq Q$ and also that the operator $K$ is contracting. From the definition of the operator $K$, it is seen that its fixed point is an ultimate solution of equation (2.11) which has the property $\lim _{t \rightarrow \infty} x(t)=1$. Hence, under the assumptions imposed, condition $(2.8)$ is not only sufficient but also necessary for equation (2.11) to have the property $\mathrm{A}$.

Condition 5 of Theorem 2.1 excludes the important particular case of equations with integrals of Volterra type.

In order to include this case as well it is necessary to sharpen conditions 3 and 6 of Theorem 2.1. The next theorem gives one of the possible variants of sharpening conditions 3 and 6 of Theorem 2.1 .

THEOREM 2.3. Let the following conditions be fulfilled:

1. Conditions $1 \mathrm{~L}-3 \mathrm{~L}, 1 \mathrm{I}, 1 \mathrm{~K}$ and conditions 2 and 4 of Theorem 2.1 hold.

2. There exists a point $t_{k} \in \mathbf{R}$ such that $\operatorname{sgn} K(t, s, u)=\operatorname{sgn} u$ for all points $(t, s, u) \in D_{K}$ such that $t \geq t_{k}$.

3. For any constant $M>0$ the following relation holds

$$
\sup _{\substack{|s|+|u| \leq M \\(t, s, u) \in D_{K}}}|K(t, s, u)|<\infty
$$

4. The following relation holds

$$
\lim _{T \rightarrow \infty}\left(T^{-1} \int_{E} \operatorname{meas}\left\{t \in[\gamma, T] \mid s \in I_{t}\right\} d s=\infty .\right.
$$

5. For each function $\varphi \in D$ there exists a number $\gamma_{\varphi} \geq \gamma$ such that $I_{t} \subseteq\left[\alpha_{\varphi}, \infty\right)$ for $t \in\left[\gamma_{\varphi}, \infty\right)$. Then each ultimate solution $X$ of equation (1.3) either oscillates or has the property $\liminf _{t \rightarrow \infty}|(L x)(t)|=0$.

PROOF. It is not difficult to see that the operator $F$ defined by equality in general does not satisfy condition $1 \mathrm{~F}$.

That is why for $n \geq 2$ we shall apply Theorem 1 to the equation

$$
[(L x)(t)]^{(n-1)}+\int_{\delta_{x}}^{t}\left(\int_{I_{t}} K(\tau, s, x,(s)) d s\right) d \tau-\left.[(L x)(t)]^{(n-1)}\right|_{t=\delta_{x}}=0
$$

where $\delta_{x}$ is an arbitrary sufficiently large number.

Assume that $D$ consists of only one ultimate solution of equation (1.1) and that this solution $x$ is non-oscillating and $\liminf _{t \rightarrow \infty}|(L x)(t)|>0$.

We shall prove that the following relation holds

$$
\lim _{T \rightarrow \infty} \int_{\delta_{x}}^{T}\left(\int_{I_{t}}|K(t, s, x(s))| d s\right) d t=\infty
$$


Without loss of generality we can assume that $x$ is an ultimately positive solution and represent the integral in (2.13) in the form

$$
\begin{aligned}
\int_{\delta_{x}}^{T}\left(\int_{I_{t}}|K(t, s, x(s))| d s\right) d t=T\left\{T^{-1} \int_{\delta_{x}}^{T}\left(\int_{I_{t} \cap\left[\delta_{x}, \infty\right)}|K(t, s, x(s))| d s\right) d t\right. \\
\left.+T^{-1} \int_{\delta_{x}}^{T}\left(\int_{I_{t} \backslash\left[\delta_{x}, \infty\right)}|K(t, s, x(s))| d s\right) d t\right\}
\end{aligned}
$$

The first integral in the right-hand side of equality (2.14) for sufficiently large $\delta_{x}$ can be estimated from below as the integral in (2.2) in the proof of Theorem (2.1) and, in view of condition 4 of Theorem 2.3, we conclude that

$$
\lim _{T \rightarrow \infty}\left(T^{-1} \int_{\delta_{x}}^{T} \int_{I_{t} \cap\left[\delta_{x}, \infty\right)}|K(t, s, x(s))| d s d t\right)=\infty
$$

From condition $1 \mathrm{I}$ and conditions 3 and 5 of Theorem 2.3 it follows that the second integral in the right-hand side of equality (2.14) for $T \rightarrow \infty$ is of order $O(T)$ and, in view of equalities (2.14) and (2.15), we conclude that relation (2.13) holds.

From condition $1 \mathrm{~K}$, condition 2 of Theorem 2.3 and (2.13) it follows that for $n \geq 2$ we can apply Theorem 1 to equation (2.12), hence each of its solutions either oscillates, or $\lim _{t \rightarrow \infty}(L x)(t)=0$ which contradicts our assumption concerning $x(t)$.

In the case $n=1$ the contradiction follows immediately from (2.12) and (2.13).

This completes the proof of Theorem 2.3.

COROLLARY 2.1. Let the following conditions be fulfilled:

1. Conditions 1 and 2 of Lemma 2.1 and conditions $1 \mathrm{I}$ and $1 \mathrm{~K}$ hold.

2. Condition 4 of Theorem 2.1, condition 3 of Theorem 2.2 and conditions 2, 3, and 5 of Theorem 2.3 hold.

3. The following relation holds

$$
\lim _{T \rightarrow \infty}\left(T^{-1} \int_{\gamma}^{\infty} \underset{s \leq \sigma \leq s+6 \tau}{\operatorname{vrainf}} \operatorname{meas}\left\{t \in[\gamma, T] \mid \sigma \in I_{t}\right\} d s\right)=\infty .
$$

Then each solution $x$ of equation (2.7) either oscillates or $\lim _{t \rightarrow \infty}|(L x)(t)|=0$.

The proof of Corollary 2.1 follows immediately from Lemmas 2.1 and 2.2 and Theorem 2.3.

It is immediately seen that Remark 2.7 still holds for relation (2.16). In the case when $I_{t}$ is of the form (2.9), the functions $\alpha(t)$ and $\beta(t)$ are continuous and $\sup _{t \in[t}|\alpha(t)|<\infty$, condition (2.16) takes the following simple form

$$
\lim _{T \rightarrow \infty}\left(T^{-1} \int_{t_{o}}^{T} \beta(t) d t\right)=\infty
$$

We shall note that in the work the properties of the solutions of equations (1.3) and (2.7) are investigated, the existence of which is assumed a priori.

This makes possible a trivial generalization of the results obtained for the case when the set $I_{t}$ and the function $K$ depend on the solution $x$. For instance, if $x=x^{*}(t)$ is a solution of an equation 
of the form (1.3) with $I=I_{t, x(t)}$, then the same function is a solution for $I=I_{t, x^{*}}(t)$ as well, whence our assertion follows. Generally speaking, the general scheme of such a transition is the following: if a set $M$ and a map $\Phi: M \rightarrow 2^{p}, P \subseteq M$, are given, then $x \in \Phi(x)$ implies $x \in P$. In our case $M$ is the set of the functional parameters which can participate in equation (1.3), $\Phi$ is the set of the respective solutions and $P$ is the set of the solutions having the property $\mathrm{A}$.

\section{REFERENCES}

1. NORKIN, S.B., Oscillation of the Solutions of Differential Equations with Deviating Argument, In the Book "Differential Equations with Deviating Argument", Nauvoka dumka, Kiev, (1977) (247-256) (in Russian).

2. MYSHKIS, A.D., BAINOV, D.D., ZAHARIEV, A.I., Oscillatory and Asymptotic Properties of a Class of Operator-Differential Inequalities, Proc. Roy. Soc. Edingurgh 96A (1984) 5-13.

3. BAINOV, D.D., ZAHARIEV, A.I., MYSHKIS, A.D., Oscillatory Properties of the Solutions of a Class of Integro-Differential Equations of Neutral Type, In the book "IX ICNO", Naukova dumka, Kiev, 1984, Vol. 2, 39-40 (in Russian).

4. BAINOV, D.D., MYSHKIS, A.D., ZAHARIEV, A.I., Asymptotic and Oscillatory Properties of a Class of Operator-Differential Inequalities, Annali di Mat. Pura ed Appl. (IV). Vol CXLIII, 1986 (197-205).

5. ZAHARIEV, A.I., BAINOV, D.D., Oscillating Properties of the Solutions of a Class of Neutral Type Functional Differential Equations, Bull. Austral. Math. Soc., Vol. 22 (1980) (365-372).

6. ZAHARIEV, A.I., BAINOV, D.D., Oscillating and Asymptotic Properties of a Class of Functional Differential Equations with Maxima, Czechoslovak Math. J.. 34 (109), (1984) (247-251).

7. BAINOV, .D.D., MYSHKIS, A.D., ZAHARIEV, A.I., Oscillating Properties of the Solutions of a Class of Neutral Type Integro-Differential Equations, Bull of the Institute of Math. Academia Sinica, Vol. 12, No. 4, (1984) (337-342). 


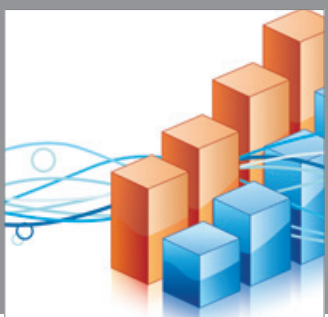

Advances in

Operations Research

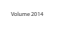

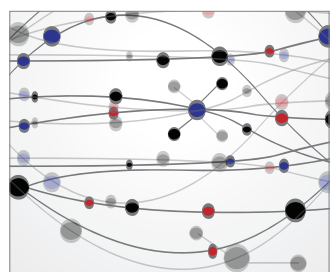

\section{The Scientific} World Journal
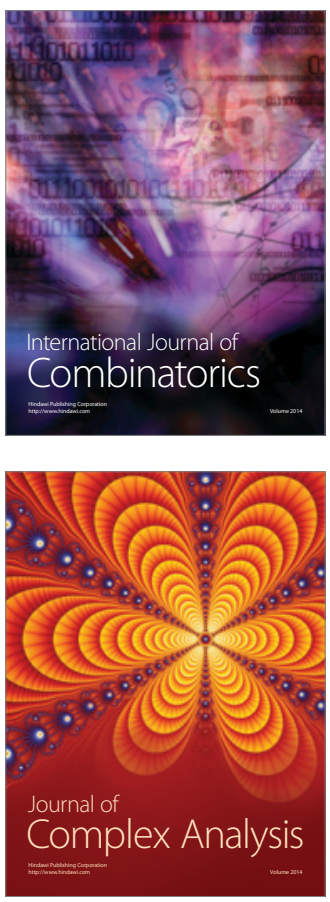

International Journal of

Mathematics and

Mathematical

Sciences
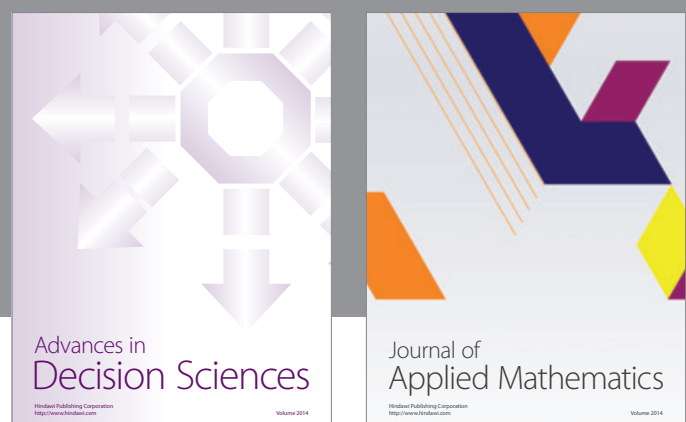

Journal of

Applied Mathematics
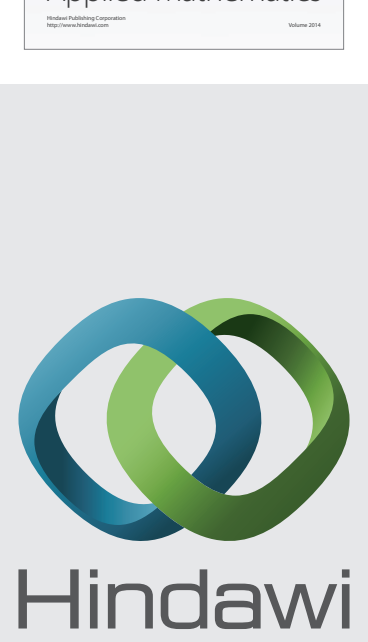

Submit your manuscripts at http://www.hindawi.com
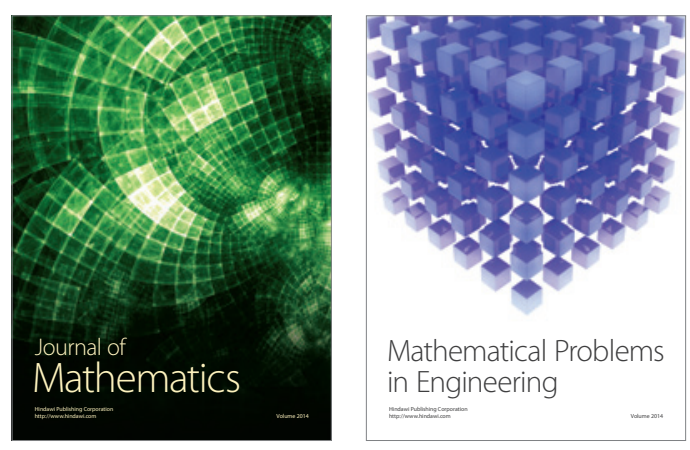

Mathematical Problems in Engineering
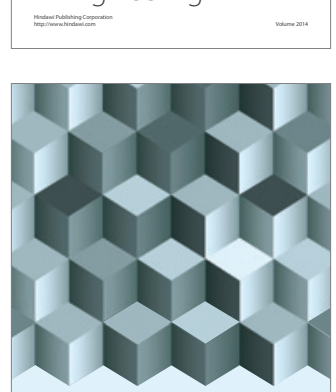

Journal of

Function Spaces
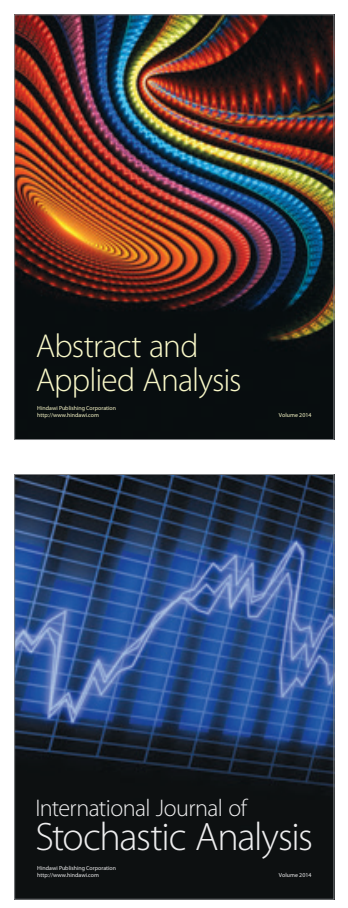

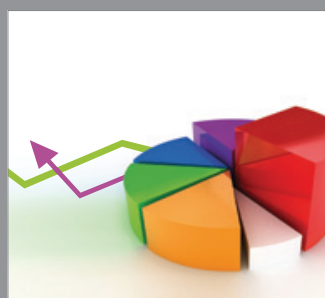

ournal of

Probability and Statistics

Promensencen
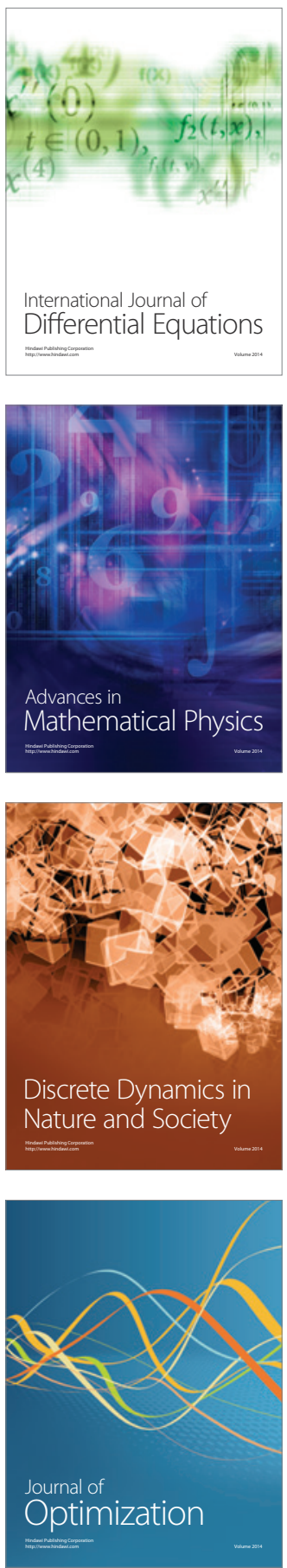